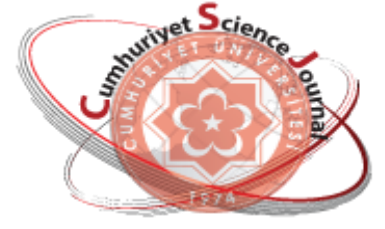

e-ISSN: $2587-246 X$

ISSN: 2587-2680

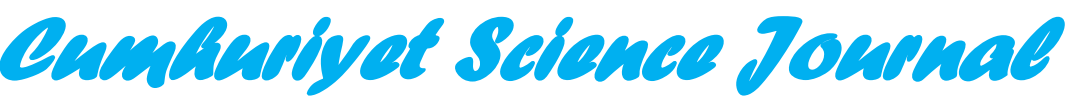

CST

Cumhuriyet Sci. J., Vol.39-3(2018) 597-607

\title{
Computation of Trace and Nodal Points of Eigenfunctions for a Sturm- Liouville Problem with Retarded Argument
}

\author{
Erdoğan ŞEN
}

Tekirdag Namik Kemal University, Faculty of Arts and Science, Department of Mathematics, Tekirdă̆, TURKEY

Received: 18.04.2018; Accepted: 28.09.2018

http://dx.doi.org/10.17776/csj.416602

\begin{abstract}
In this study, a formula for regularized sums of eigenvalues and nodal points of eigenfunctions for a discontinuous Sturm-Liouville problem with a constant retarded argument. Contrary to standart problems the spectral parameter appears not only in the differential equation, but also in one of the boundary conditions. Thus, we see whether the nodal points of eigenfunctions and the trace change or not.
\end{abstract}

Keywords: Differential equation with retarded argument, transmission conditions, spectrum, nodal points, regularized trace.

\section{Geciken Argümanlı Bir Sturm-Liouville Probleminin İzinin ve Özfonksiyonlarının Düğüm Noktalarının Hesaplanması}

Özet. Biz bu çalışmada sabit geciken argümanlı süreksiz bir Sturm-Liouville probleminin özdeğerleri için bir iz formülü ve özfonksiyonları için düğüm noktaları elde ettik. Standart problemlerin aksine spektral parametre sadece diferansiyel denklemde değil ayrıca sınır koşullarının birinde de yer alır. Böylece bu durumun özfonksiyonların düğüm noktalarını ve izini değiştirip değiştirmediğini görmüş oluyoruz.

Anahtar Kelimeler: Geciken argümanlı diferansiyel denklem, geçiş koşulları, spectrum, düğüm noktaları, düzenli iz.

\section{INTRODUCTION}

Differential equations with retarded argument is an active research area of delay differential equations. Each year there is an increase in the number of articles devoted to the study of various applied problems formulated with the use of delays. However, in an overwhelming majority of applied articles, constant delays are considered. Such a consideration is an improvement compared with the model of an "ideal" process which is obtained if it is assumed that there are no delays at all, that the "functioning" takes place instantly. For linear equations with constant delay, very effective operational methods are available (e.g., first of all the Laplace transform) [1].

To deal with interior discontinuities, some conditions are imposed on the discontinuous points, which are often called transmission conditions (see [2-8]), interface conditions (see [9]), or point interactions (see [10]). The problems with transmission conditions have become an important area of research in recent years because of the needs of modern technology, engineering, and physics. In this paper, we consider the boundary-value-transmission problem for the differential equation with a constant retarded argument

$$
a(t) y^{\prime \prime}(t)+q(t) y(t-\theta)+\rho^{2} y(t)=0,
$$

\footnotetext{
* Corresponding author. Email address: esen@nku.edu.tr

http://dergipark.gov.tr/csj C2016 Faculty of Science, Cumhuriyet University
} 
on $\Lambda=\left[0, \frac{\pi}{2}\right) \cup\left(\frac{\pi}{2}, \pi\right]$, with boundary conditions

$$
\begin{gathered}
y(0)+\rho y^{\prime}(0)=0, \\
y^{\prime}(\pi)=0
\end{gathered}
$$

and transmission conditions

$$
\begin{aligned}
& y\left(\frac{\pi}{2}-0\right)=\delta y\left(\frac{\pi}{2}+0\right), \\
& y^{\prime}\left(\frac{\pi}{2}-0\right)=\delta y^{\prime}\left(\frac{\pi}{2}+0\right),
\end{aligned}
$$

where $a(t)=a_{1}^{2}$ for $t \in \Lambda^{-}=\left[0, \frac{\pi}{2}\right)$ and $a(t)=a_{2}^{2}$ for $t \in \Lambda^{+}=\left(\frac{\pi}{2}, \pi\right]$; the real-valued function $q(t)$ is continuous in $\Lambda$ and has finite limits $q\left(\frac{\pi}{2} \pm 0\right)=\lim _{t \rightarrow \frac{\pi}{2} \pm 0} q(t), \theta$ is a non-negative constant such that, if $t \in \Lambda^{-}$then $t-\theta \geq 0$; if $t \in \Lambda^{+}$then $t-\theta \geq \frac{\pi}{2} ; \rho$ is a spectral parameter; $\delta$ and $a_{i}$ 's $(i=1,2)$ are arbitrary real numbers such that $a_{i} \neq 0 \quad(i=1,2)$.

The goal of this article is to calculate the regularized trace for the problem (1)-(5). We point out that our results are extension and/or generalization to those in [11-21]. For example, if the retardation $\theta \equiv 0$ in (1) and $a(t) \equiv 1, \delta=1$ then we have the formula of the first regularized trace for the classical SturmLiouville operator which is called Gelfand-Levitan formula (see [12]). We also want to emphasize that the problem studied here differs from the standard boundary value problems in that it contains transmission conditions and the spectral parameter appears not only in the differential equation, but also in the boundary conditions. Because, it is important in the literature to know when the spectral parameter is both in the differential equation and one of the boundary conditions, whether the asymptotic formulas of eigenvalues and the trace change or not.

\section{THE SPECTRUM}

Let $\omega_{1}(t, \rho)$ be a solution of Eq. (1) on $\overline{\Lambda^{-}}=\left[0, \frac{\pi}{2}\right]$, satisfying the initial conditions

$$
\omega_{1}(0, \rho)=1 \text { and } \omega_{1}^{\prime}(0, \rho)=-1 \text {. }
$$

The conditions (6) define a unique solution of Eq. (1) on $\overline{\Lambda^{-}}$(see $\left.[1,22]\right)$.

After defining the above solution, then we will define the solution $\omega_{2}(t, \rho)$ of Eq. (1) on $\overline{\Lambda^{+}}=\left[\frac{\pi}{2}, \pi\right]$ by means of the solution $\omega_{1}(t, \rho)$ using the initial conditions

$$
\omega_{2}\left(\frac{\pi}{2}, \rho\right)=\delta^{-1} \omega_{1}\left(\frac{\pi}{2}, \rho\right), \quad \omega_{2}^{\prime}\left(\frac{\pi}{2}, \rho\right)=\delta^{-1} \omega_{1}^{\prime}\left(\frac{\pi}{2}, \rho\right) .
$$

The conditions (7) define a unique solution of Eq. (1) on $\overline{\Lambda^{+}}$(see [22]).

Consequently, the function $\omega(t, \rho)$ is defined on $\Lambda$ by the equality

$$
\omega(t, \rho)= \begin{cases}\omega_{1}(t, \rho), & t \in \Lambda^{-}, \\ \omega_{2}(t, \rho), & t \in \Lambda^{+}\end{cases}
$$


is a solution of (1) on $\Lambda$, which satisfies one of the boundary conditions and the interface conditions (4)-(5) Then the following integral equations hold:

$$
\begin{gathered}
\omega_{1}(t, \rho)=\rho \cos \frac{\rho}{a_{1}} t-\frac{a_{1}}{\rho} \sin \frac{\rho}{a_{1}} t \\
-\frac{1}{\rho a_{1}} \int_{0}^{t} q(\tau) \sin \frac{\rho}{a_{1}}(t-\tau) \omega_{1}(\tau-\theta, \rho) d \tau \\
\omega_{2}(t, \rho)=\frac{1}{\delta} \omega_{1}\left(\frac{\pi}{2}, \rho\right) \cos \frac{\rho}{a_{2}}\left(t-\frac{\pi}{2}\right)+\frac{a_{2} \omega_{1}^{\prime}\left(\frac{\pi}{2}, \rho\right)}{\rho \delta} \sin \frac{\rho}{a_{2}}\left(t-\frac{\pi}{2}\right) \\
-\frac{1}{\rho a_{2}} \int_{\frac{\pi}{2}}^{t} q(\tau) \sin \frac{\rho}{a_{2}}(t-\tau) \omega_{2}(\tau-\theta, \rho) d \tau .
\end{gathered}
$$

Solving the equations (8)-(9) by the method of successive approximation, we obtain the following asymptotic equalities for $|\rho| \rightarrow \infty$ :

$$
\begin{aligned}
& \omega_{1}(t, \rho)=\rho \cos \frac{\rho}{a_{1}} t-\frac{a_{1}}{\rho} \sin \frac{\rho}{a_{1}} t-\frac{1}{2 a_{1}} \int_{0}^{t} q(\tau) \sin \frac{\rho}{a_{1}}(t-\theta) d \tau \\
& -\frac{1}{2 a_{1}} \int_{0}^{t} q(\tau) \sin \frac{\rho}{a_{1}}(t-(2 \tau-\theta)) d \tau+O\left(\frac{1}{\rho^{2}}\right) .
\end{aligned}
$$

Differentiating (10) with respect to $t$, we get

$$
\begin{gathered}
\omega_{1}^{\prime}(t, \rho)=-\frac{\rho^{2}}{a_{1}} \sin \frac{\rho}{a_{1}} t-\cos \frac{\rho}{a_{1}} t-\frac{\rho}{2 a_{1}^{2}} \int_{0}^{t} q(\tau) \cos \frac{\rho}{a_{1}}(t-\theta) d \tau \\
-\frac{\rho}{2 a_{1}^{2}} \int_{0}^{t} q(\tau) \cos \frac{\rho}{a_{1}}(t-(2 \tau-\theta)) d \tau+O\left(\frac{1}{\rho}\right) .
\end{gathered}
$$

Using the fact that,

$$
O\left(\frac{1}{\rho}\right)=\left\{\begin{array}{l}
\int_{0}^{t} q(\tau) \sin \frac{\rho}{a_{1}}(2 \tau-\theta) d \tau, \quad t \in \overline{\Lambda^{-}} ; \\
\int_{0}^{t} q(\tau) \cos \frac{\rho}{a_{1}}(2 \tau-\theta) d \tau, \quad t \in \overline{\Lambda^{-}} \\
\int_{\frac{\pi}{2}}^{t} q(\tau) \sin \frac{\rho}{a_{2}}(2 \tau-\theta) d \tau, \quad t \in \overline{\Lambda^{+}} ; \\
\int_{\frac{\pi}{2}}^{t} q(\tau) \cos \frac{\rho}{a_{2}}(2 \tau-\theta) d \tau, \quad t \in \overline{\Lambda^{+}}
\end{array}\right.
$$

(see [1]), (10) and (11) we have

$$
\begin{gathered}
\omega_{2}(t, \rho)=\frac{\rho}{\delta} \cos \frac{\rho}{a_{2}}\left(\frac{\pi\left(a_{2}-a_{1}\right)}{2 a_{1}}+t\right)-\frac{a_{1}}{\rho \delta} \sin \frac{\rho}{a_{2}}\left(\frac{\pi\left(a_{2}-a_{1}\right)}{2 a_{1}}+t\right) \\
-\frac{\left(a_{1}+a_{2}\right)}{2 \delta a_{1} a_{2}}\left\{\left[B\left(\frac{\pi}{2}, \rho, \theta\right)+D(t, \rho, \theta)\right] \sin \frac{\rho}{a_{2}}\left(\frac{\pi\left(a_{2}-a_{1}\right)}{2 a_{1}}+t\right)\right.
\end{gathered}
$$




$$
\left.+\left[A\left(\frac{\pi}{2}, \rho, \theta\right)+C(t, \rho, \theta)\right] \cos \frac{\rho}{a_{2}}\left(\frac{\pi\left(a_{2}-a_{1}\right)}{2 a_{1}}+t\right)\right\}+O\left(\frac{1}{\rho^{2}}\right)
$$

Here,

$$
\begin{aligned}
& A(t, \rho, \theta)=\int_{0}^{t} q(\tau) \sin \frac{\rho \theta}{a_{1}} d \tau \quad t \in \overline{\Lambda^{-}} ; \quad B(t, \rho, \theta)=\int_{0}^{t} q(\tau) \cos \frac{\rho \theta}{a_{1}} d \tau \quad t \in \overline{\Lambda^{-}} \\
& C(t, \rho, \theta)=\int_{\frac{\pi}{2}}^{t} q(\tau) \sin \frac{\rho \theta}{a_{2}} d \tau, \quad t \in \overline{\Lambda^{+}} ; \quad D(t, \rho, \theta)=\int_{\frac{\pi}{2}}^{t} q(\tau) \cos \frac{\rho \theta}{a_{2}} d \tau, \quad t \in \overline{\Lambda^{+}}
\end{aligned}
$$

Differentiating (12) with respect to $t$, we get

$$
\begin{gathered}
\omega_{2}^{\prime}(t, \rho)=-\frac{\rho^{2}}{\delta a_{2}} \sin \frac{\rho}{a_{2}}\left(\frac{\pi\left(a_{2}-a_{1}\right)}{2 a_{1}}+t\right)-\frac{a_{1}}{\delta a_{2}} \cos \frac{\rho}{a_{2}}\left(\frac{\pi\left(a_{2}-a_{1}\right)}{2 a_{1}}+t\right) \\
-\frac{\rho\left(a_{1}+a_{2}\right)}{2 \delta a_{1} a_{2}^{2}}\left\{\left[B\left(\frac{\pi}{2}, \rho, \theta\right)+D(t, \rho, \theta)\right] \cos \frac{\rho}{a_{2}}\left(\frac{\pi\left(a_{2}-a_{1}\right)}{2 a_{1}}+t\right)\right. \\
\left.-\left[A\left(\frac{\pi}{2}, \rho, \theta\right)+C(t, \rho, \theta)\right] \sin \frac{\rho}{a_{2}}\left(\frac{\pi\left(a_{2}-a_{1}\right)}{2 a_{1}}+t\right)\right\}+O\left(\frac{1}{\rho}\right) .
\end{gathered}
$$

The solution $\omega(t, \rho)$ defined above is a nontrivial solution of (1) satisfying conditions (2) and (4)-(5). Putting $\omega(t, \rho)$ into (3), we get the characteristic equation

$$
\Theta(\rho) \equiv \omega^{\prime}(\pi, \rho)=0 .
$$

The set of eigenvalues of boundary value problem (1)-(5) coincides with the set of the squares of roots of (14), and eigenvalues are simple (see [23]). From (12), (13) and (14), we obtain

$$
\begin{aligned}
& \Theta(\rho) \equiv-\frac{\rho^{2}}{\delta a_{2}} \sin \frac{\rho \pi}{a_{2}}\left(\frac{a_{2}-a_{1}}{2 a_{1}}+1\right)-\frac{a_{1}}{\delta a_{2}} \cos \frac{\rho \pi}{a_{2}}\left(\frac{a_{2}-a_{1}}{2 a_{1}}+1\right) \\
& -\frac{\rho\left(a_{1}+a_{2}\right)}{2 \delta a_{1} a_{2}^{2}}\left\{\left[B\left(\frac{\pi}{2}, \rho, \theta\right)+D(\pi, \rho, \theta)\right] \cos \frac{\rho \pi}{a_{2}}\left(\frac{a_{2}-a_{1}}{2 a_{1}}+1\right)\right. \\
& \left.-\left[A\left(\frac{\pi}{2}, \rho, \theta\right)+C(\pi, \rho, \theta)\right] \sin \frac{\rho \pi}{a_{2}}\left(\frac{a_{2}-a_{1}}{2 a_{1}}+1\right)\right\}+O\left(\frac{1}{\rho}\right) .
\end{aligned}
$$

Define

$$
\Theta_{0}(\rho) \equiv-\frac{\rho^{2}}{\delta a_{2}} \sin \frac{\rho \pi}{a_{2}}\left(\frac{a_{2}-a_{1}}{2 a_{1}}+1\right)
$$

Denote by $\rho_{n}^{0}=\frac{2 a_{1} a_{2} n}{a_{1}+a_{2}}, n \in \mathrm{Z}$, zeros of the function $\Theta_{0}(\rho)$. It is simple algebraically except for $\rho_{ \pm 0}^{0}$ and we have

$$
\rho_{n} \sim \rho_{n}^{0}+O\left(\frac{1}{n}\right)
$$


Denote by $C_{n}$ the circle of radius, $0<\varepsilon<\frac{1}{2}$, centered at the origin $\rho_{n}^{0}$ and by $\Gamma_{N_{0}}$ the counterclockwise square contours with four vertices

$$
\begin{gathered}
K=N_{0}+\varepsilon+N_{0} i, \quad L=-N_{0}-\varepsilon+N_{0} i, \\
M=-N_{0}-\varepsilon-N_{0} i, \quad N=N_{0}+\varepsilon-N_{0} i,
\end{gathered}
$$

where $i=\sqrt{-1}$ and $N_{0}$ is a natural number. Obviously, if $\rho \in C_{n}$ or $\rho \in \Gamma_{N_{0}}$, then $\left|\Theta_{0}(\rho)\right| \geq M|\rho| e^{|\operatorname{Im} \rho| \pi} \quad(M>0)$ by using a similar method in [16]. Thus, on $\rho \in C_{n}$ or $\rho \in \Gamma_{N_{0}}$, we have

$$
\begin{gathered}
\frac{\Theta(\rho)}{\Theta_{0}(\rho)}=1+\frac{a_{1}+a_{2}}{2 \rho a_{1} a_{2}} \\
\times\left[\left(B\left(\frac{\pi}{2}, \rho, \theta\right)+D(\pi, \rho, \theta)\right) \cot \frac{\rho \pi}{a_{2}}\left(\frac{a_{2}-a_{1}}{2 a_{1}}+1\right)-\left(A\left(\frac{\pi}{2}, \rho, \theta\right)+C(\pi, \rho), \theta\right)\right]+O\left(\frac{1}{\rho^{2}}\right) .
\end{gathered}
$$

Expanding $\ln \frac{\Theta(\rho)}{\Theta_{0}(\rho)}$ by the Maclaurin formula, we find that

$$
\begin{gathered}
\ln \frac{\Theta(\rho)}{\Theta_{0}(\rho)}=\frac{1}{\rho}\left\{\frac { a _ { 1 } + a _ { 2 } } { 2 a _ { 1 } a _ { 2 } } \left[\left(B\left(\frac{\pi}{2}, \rho, \theta\right)+D(\pi, \rho, \theta)\right) \cot \frac{\rho \pi}{a_{2}}\left(\frac{a_{2}-a_{1}}{2 a_{1}}+1\right)\right.\right. \\
\left.\left.-\left(A\left(\frac{\pi}{2}, \rho, \theta\right)+C(\pi, \rho, \theta)\right)\right]\right\} \\
-\frac{1}{2 \rho^{2}}\left\{\left[\frac{a_{1}+a_{2}}{2 a_{1} a_{2}}\left(B\left(\frac{\pi}{2}, \rho, \theta\right)+D(\pi, \rho, \theta)\right)\right]^{2} \cot ^{2} \frac{\rho \pi}{a_{2}}\left(\frac{a_{2}-a_{1}}{2 a_{1}}+1\right)\right. \\
+\frac{\left(a_{1}+a_{2}\right)^{2}}{4 a_{1}^{2} a_{2}^{2}}\left(A\left(\frac{\pi}{2}, \rho, \theta\right)+C(\pi, \rho, \theta)\right)^{2} \\
+\frac{a_{1}+a_{2}}{a_{1} a_{2}}\left(B\left(\frac{\pi}{2}, \rho, \theta\right)+D(\pi, \rho, \theta)\right)\left(A\left(\frac{\pi}{2}, \rho, \theta\right)+C(\pi, \rho, \theta)\right) \\
\left.\times \cot \frac{\rho \pi}{a_{2}}\left(\frac{a_{2}-a_{1}}{2 a_{1}}+1\right)\right\}+O\left(\frac{1}{\rho^{3}}\right) .
\end{gathered}
$$

Using the well-known Rouche Theorem, we get that $\Theta(\rho)$ has the same number of zeros inside $\Gamma_{N_{0}}$ as $\Theta_{0}(\rho)$ (see [24]). Using the residue theorem, we have

$$
\begin{gathered}
\rho_{n}-\rho_{n}^{0}=-\frac{1}{2 \pi i} \oint_{C_{n}} \ln \frac{\Theta(\rho)}{\Theta_{0}(\rho)} d \rho \\
=-\frac{1}{2 \pi i} \oint_{C_{n}}\left[\frac{a_{1}+a_{2}}{2 a_{1} a_{2}}\left(B\left(\frac{\pi}{2}, \rho, \theta\right)+D(\pi, \rho, \theta)\right)\right] \frac{\cot \frac{\rho \pi}{a_{2}}\left(\frac{a_{2}-a_{1}}{2 a_{1}}+1\right)}{\rho} d \rho
\end{gathered}
$$




$$
\begin{gathered}
+\frac{1}{2 \pi i} \oint_{C_{n}} \frac{a_{1}+a_{2}}{2 a_{1} a_{2}}\left(A\left(\frac{\pi}{2}, \rho, \theta\right)+C(\pi, \rho, \theta)\right) \frac{d \rho}{\rho} \\
+\frac{1}{2 \pi i} \oint_{C_{n}}\left[\frac{a_{1}+a_{2}}{2 a_{1} a_{2}}\left(B\left(\frac{\pi}{2}, \rho, \theta\right)+D(\pi, \rho, \theta)\right)\right]^{2} \frac{\cot ^{2} \frac{\rho \pi}{a_{2}}\left(\frac{a_{2}-a_{1}}{2 a_{1}}+1\right)}{2 \rho^{2}} d \rho \\
+\frac{1}{2 \pi i} \oint_{C_{n}} \frac{\left(a_{1}+a_{2}\right)^{2}}{4 a_{1}^{2} a_{2}^{2}}\left(A\left(\frac{\pi}{2}, \rho, \theta\right)+C(\pi, \rho, \theta)\right)^{2} \frac{d \rho}{2 \rho^{2}} \\
+\frac{1}{2 \pi i} \oint_{C_{n}} \frac{a_{1}+a_{2}}{a_{1} a_{2}}\left[\frac{a_{1}+a_{2}}{2 a_{1} a_{2}}\left(B\left(\frac{\pi}{2}, \rho, \theta\right)+D(\pi, \rho, \theta)\right)\right] \\
\times\left(A\left(\frac{\pi}{2}, \rho, \theta\right)+C(\pi, \rho, \theta)\right) \frac{\cot \frac{\rho \pi}{a_{2}}\left(\frac{a_{2}-a_{1}}{2 a_{1}}+1\right)}{2 \rho^{2}} d \rho+O\left(\frac{1}{n^{3}}\right) .
\end{gathered}
$$

Thus, using (15) and residue calculation we have proven the following theorem.

Theorem 2.1. The spectrum of the problem (1)-(5) has the

$$
\begin{gathered}
\rho_{n}=\rho_{n}^{0}-\frac{1}{\rho_{n}^{0} \pi}\left[\frac{a_{1}+a_{2}}{2 a_{1} a_{2}}\left(B\left(\frac{\pi}{2}, \rho_{n}^{0}, \theta\right)+D\left(\pi, \rho_{n}^{0}, \theta\right)\right)\right] \\
+\frac{1}{\left(\rho_{n}^{0}\right)^{2} \pi}\left\{\frac{a_{1}+a_{2}}{a_{1} a_{2}}\left(A\left(\frac{\pi}{2}, \rho_{n}^{0}, \theta\right)+C\left(\pi, \rho_{n}^{0}, \theta\right)\right)\left[\frac{a_{1}+a_{2}}{2 a_{1} a_{2}}\left(B\left(\frac{\pi}{2}, \rho_{n}^{0}, \theta\right)+D\left(\pi, \rho_{n}^{0}, \theta\right)\right)\right]\right\} \\
-\frac{1}{\left(\rho_{n}^{0}\right)^{3}}\left[\frac{a_{1}+a_{2}}{2 a_{1} a_{2}}\left(B\left(\frac{\pi}{2}, \rho_{n}^{0}, \theta\right)+D\left(\pi, \rho_{n}^{0}, \theta\right)\right)\right]^{2}+O\left(\frac{1}{n^{3}}\right)
\end{gathered}
$$

asymptotic distribution for sufficiently large $|n|$.

\section{THE REGULARIZED TRACE FORMULA}

In this section, we will get regularized trace formula for the problem (1)-(5).

The asymptotic formula (15) for the eigenvalues implies that for all sufficiently large $N_{0}$, the numbers $\rho_{n}$ with $|n| \leq N_{0}$ are inside $\Gamma_{N_{0}}$, and the numbers $\rho_{n}$ with $|n|>N_{0}$ are outside $\Gamma_{N_{0}}$. It follows that

$$
\begin{gathered}
\rho_{-0}^{2}+\rho_{0}^{2}+\sum_{0 \neq n=-N_{0}}^{N_{0}}\left(\rho_{n}^{2}-\left(\rho_{n}^{0}\right)^{2}\right)=-\frac{1}{2 \pi i} \oint_{\Gamma_{n}} 2 \rho \ln \frac{\theta(\rho)}{\theta_{0}(\rho)} d \rho \\
=-\frac{1}{2 \pi i} \oint_{\Gamma_{n}} 2\left[\frac{a_{1}+a_{2}}{2 a_{1} a_{2}}\left(B\left(\frac{\pi}{2}, \rho, \theta\right)+D(\pi, \rho, \theta)\right)\right] \\
\times \cot \frac{\rho \pi}{a_{2}}\left(\frac{a_{2}-a_{1}}{2 a_{1}}+1\right) d \rho+\frac{1}{2 \pi i} \oint_{\Gamma_{n}} 2\left[\frac{a_{1}+a_{2}}{2 a_{1} a_{2}}\left(A\left(\frac{\pi}{2}, \rho, \theta\right)+C(\pi, \rho, \theta)\right)\right] d \rho
\end{gathered}
$$




$$
\begin{aligned}
& +\frac{1}{2 \pi i} \oint_{\Gamma_{n}}\left[\frac{a_{1}+a_{2}}{2 a_{1} a_{2}}\left(B\left(\frac{\pi}{2}, \rho, \theta\right)+D(\pi, \rho, \theta)\right)\right]^{2} \frac{\cot ^{2} \frac{\rho \pi}{a_{2}}\left(\frac{a_{2}-a_{1}}{2 a_{1}}+1\right)}{\rho} d \rho \\
& +\frac{1}{2 \pi i} \oint_{\Gamma_{n}} \frac{\left(a_{1}+a_{2}\right)^{2}}{4 a_{1}^{2} a_{2}^{2}}\left(A\left(\frac{\pi}{2}, \rho, \theta\right)+C(\pi, \rho, \theta)\right)^{2} \frac{d \rho}{\rho} \\
& +\frac{1}{2 \pi i} \oint_{\Gamma_{n}} \frac{a_{1}+a_{2}}{a_{1} a_{2}}\left[\frac{a_{1}+a_{2}}{2 a_{1} a_{2}}\left(B\left(\frac{\pi}{2}, \rho, \theta\right)+D(\pi, \rho, \theta)\right)\right] \\
& \times\left(A\left(\frac{1}{2},,\right)+C(, \quad,)\right) \frac{\cot \frac{a_{2}}{a_{2}}\left(\frac{a_{2} a_{1}}{2 a_{1}}+1\right)}{(A)} d+O\left(\frac{1}{N_{0}}\right),
\end{aligned}
$$

by calculations, which implies that

$$
\begin{gathered}
\rho_{-0}^{2}+\rho_{0}^{2}+\sum_{0 \neq n=-N_{0}}^{N_{0}} \rho_{n}^{2}-\left(\rho_{n}^{0}\right)^{2} \\
=-\frac{2}{\pi} \sum_{0 \neq n=-N_{0}}^{N_{0}}\left[\frac{a_{1}+a_{2}}{2 a_{1} a_{2}}\left(B\left(\frac{\pi}{2}, \rho_{n}^{0}, \theta\right)+D\left(\pi, \rho_{n}^{0}, \theta\right)\right]-\frac{2}{\pi}\left[\frac{a_{1}+a_{2}}{2 a_{1} a_{2}}\left(B\left(\frac{\pi}{2}, 0, \theta\right)+D(\pi, 0, \theta)\right)\right]\right. \\
+\sum_{0 \neq n=-N_{0}}^{N_{0}} \frac{a_{1}+a_{2}}{a_{1} a_{2}}\left[\frac{a_{1}+a_{2}}{2 a_{1} a_{2}}\left(B\left(\frac{\pi}{2}, \rho_{n}^{0}, \theta\right)+D\left(\pi, \rho_{n}^{0}, \theta\right)\right)\right] \\
\times\left(A\left(\frac{\pi}{2}, \rho_{n}^{0}, \theta\right)+C\left(\pi, \rho_{n}^{0}, \theta\right)\right) \frac{1}{\rho_{n}^{0} \pi}+R \\
-\left[\frac{a_{1}+a_{2}}{2 a_{1} a_{2}}\left(B\left(\frac{\pi}{2}, 0, \theta\right)+D(\pi, 0, \theta)\right)\right]^{2}+\frac{\left(a_{1}+a_{2}\right)^{2}}{4 a_{1}^{2} a_{2}^{2}}\left(A\left(\frac{\pi}{2}, 0, \theta\right)+C(\pi, 0, \theta)\right)^{2}+O\left(\frac{1}{N_{0}}\right)
\end{gathered}
$$

where,

$$
\begin{aligned}
R= & \operatorname{Re} s_{\rho=0}\left\{\frac{a_{1}+a_{2}}{a_{1} a_{2}}\left[\frac{a_{1}+a_{2}}{2 a_{1} a_{2}}\left(B\left(\frac{\pi}{2}, \rho, \theta\right)+D(\pi, \rho, \theta)\right)\right]\right. \\
& \left.\times\left(A\left(\frac{\pi}{2}, \rho, \theta\right)+C(\pi, \rho, \theta)\right) \frac{\cot \frac{\rho \pi}{a_{2}}\left(\frac{a_{2}-a_{1}}{2 a_{1}}+1\right)}{\rho} d \rho\right\} .
\end{aligned}
$$

Passing to the limit as $N_{0} \rightarrow \infty$ in (16), we have

$$
\begin{gathered}
\rho_{-0}^{2}+\rho_{0}^{2}+\sum_{0 \neq n=-\infty}^{\infty}\left\{\rho_{n}^{2}-\left(\rho_{n}^{0}\right)^{2}+\frac{2}{\pi}\left[\frac{a_{1}+a_{2}}{2 a_{1} a_{2}}\left(B\left(\frac{\pi}{2}, \rho_{n}^{0}, \theta\right)+D\left(\pi, \rho_{n}^{0}, \theta\right)\right)\right]\right. \\
-\frac{a_{1}+a_{2}}{a_{1} a_{2}}\left[\frac{a_{1}+a_{2}}{2 a_{1} a_{2}}\left(B\left(\frac{\pi}{2}, \rho_{n}^{0}, \theta\right)+D\left(\pi, \rho_{n}^{0}, \theta\right)\right]\left(A\left(\frac{\pi}{2}, \rho_{n}^{0}, \theta\right)+C\left(\pi, \rho_{n}^{0}, \theta\right)\right) \frac{1}{\rho_{n}^{0} \pi}\right\} \\
=-\frac{2}{\pi}\left[\frac{a_{1}+a_{2}}{2 a_{1} a_{2}}\left(B\left(\frac{\pi}{2}, 0, \theta\right)+D(\pi, 0, \theta)\right)\right]+R
\end{gathered}
$$




$$
-\left[\frac{a_{1}+a_{2}}{2 a_{1} a_{2}}\left(B\left(\frac{\pi}{2}, 0, \theta\right)+D(\pi, 0, \theta)\right)\right]^{2}+\frac{\left(a_{1}+a_{2}\right)^{2}}{4 a_{1}^{2} a_{2}^{2}}\left(A\left(\frac{\pi}{2}, 0, \theta\right)+C(\pi, 0, \theta)\right)^{2} .
$$

The series on the left side of (17) is called the regularized trace of the problem (1)-(5).

\section{THE NODAL POINTS}

In this chapter, following [16], we will find nodal points of eigenfunctions of the problem (1)-(5).

Let us rewrite the equation (10) and replace $\rho$ by $\rho_{n}$ :

$$
\begin{aligned}
\omega_{1}\left(t, \rho_{n}\right)= & \rho_{n} \cos \frac{\rho_{n} t}{a_{1}}-\frac{a_{1}}{\rho_{n}} \sin \frac{\rho_{n} t}{a_{1}}-\frac{\sin \frac{\rho_{n} t}{a_{1}}}{2 a_{1}} \int_{0}^{t} q(\tau) \cos \left(\frac{\rho_{n} \theta}{a_{1}}\right) d \tau \\
& +\frac{\cos \frac{\rho_{n} t}{a_{1}}}{2 a_{1}} \int_{0}^{t} q(\tau) \sin \left(\frac{\rho_{n} \theta}{a_{1}}\right) d \tau+O\left(\frac{1}{\rho_{n}^{2}}\right) .
\end{aligned}
$$

Let us assume that $t_{n}^{j}$ are the nodal points of the eigenfunction $\omega_{1}\left(t, \rho_{n}\right)$. Taking $\sin \left(\frac{\rho_{n} t}{a_{1}}\right) \neq 0$ into account for sufficiently large $n$, we get

$$
\cot \left(\frac{\rho_{n} t}{a_{1}}\right)\left[1+\frac{A\left(t, \rho_{n}, \theta\right)}{2 \rho_{n} a_{1}}\right]=\frac{B\left(t, \rho_{n}, \theta\right)}{2 \rho_{n} a_{1}}+O\left(\frac{1}{\rho_{n}^{2}}\right) .
$$

It follows easily that

$$
\tan \left(\frac{\rho_{n} t}{a_{1}}+\frac{\pi}{2}\right)=-\frac{B\left(t, \rho_{n}, \theta\right)}{2 \rho_{n} a_{1}}+O\left(\frac{1}{\rho_{n}^{2}}\right) .
$$

Thus, solving the equation (18), one obtains

$$
t_{n}^{j}=\frac{\left(j-\frac{1}{2}\right) \pi a_{1}}{\rho_{n}}-\frac{B\left(t_{n}^{j}, \rho_{n}, \theta\right)}{2 \rho_{n}^{2}}+O\left(\frac{1}{\rho_{n}^{3}}\right) .
$$

Note that

$$
\rho_{n}^{-1}=\frac{1}{\rho_{n}^{0}}+\frac{\left[\frac{a_{1}+a_{2}}{2 a_{1} a_{2}}\left(B\left(\frac{\pi}{2}, \rho_{n}^{0}, \theta\right)+D\left(\pi, \rho_{n}^{0}, \theta\right)\right)\right]}{\left(\rho_{n}^{0}\right)^{3} \pi}+O\left(\frac{1}{n^{4}}\right)
$$

and

$$
\rho_{n}^{-2}=\frac{1}{\left(\rho_{n}^{0}\right)^{2}}+O\left(\frac{1}{n^{4}}\right)
$$

Substituting (20) and (21) into (19) we have

$$
t_{n}^{j}=\frac{\left(j-\frac{1}{2}\right) \pi a_{1}}{\rho_{n}^{0}}-\frac{\left(j-\frac{1}{2}\right) a_{1}\left[\frac{a_{1}+a_{2}}{2 a_{1} a_{2}}\left(B\left(\frac{\pi}{2}, \rho_{n}^{0}, \theta\right)+D\left(\pi, \rho_{n}^{0}, \theta\right)\right)\right]}{\left(\rho_{n}^{0}\right)^{3}}
$$




$$
-\frac{B\left(\frac{\left(j-\frac{1}{2}\right) \pi a_{1}}{\rho_{n}^{0}}, \rho_{n}^{0}\right)}{2\left(\rho_{n}^{0}\right)^{2}}+O\left(\frac{1}{n^{3}}\right), \quad j=1, \overline{\left[\frac{n}{2}\right]} .
$$

Similarly, from (12), we get

$$
\begin{gathered}
\delta \omega_{2}\left(t, \rho_{n}\right)=\rho_{n} \cos \frac{\rho_{n}}{a_{2}}\left(\frac{\pi\left(a_{2}-a_{1}\right)}{2 a_{1}}+t\right)-\frac{a_{1}}{\rho_{n}} \sin \frac{\rho_{n}}{a_{2}}\left(\frac{\pi\left(a_{2}-a_{1}\right)}{2 a_{1}}+t\right) \\
-\frac{\left(a_{1}+a_{2}\right)}{2 a_{1} a_{2}}\left\{\left[B\left(\frac{\pi}{2}, \rho_{n}, \theta\right)+D\left(t, \rho_{n}, \theta\right)\right] \sin \frac{\rho_{n}}{a_{2}}\left(\frac{\pi\left(a_{2}-a_{1}\right)}{2 a_{1}}+t\right)\right. \\
\left.+\left[A\left(\frac{\pi}{2}, \rho_{n}, \theta\right)+C\left(t, \rho_{n}, \theta\right)\right] \cos \frac{\rho_{n}}{a_{2}}\left(\frac{\pi\left(a_{2}-a_{1}\right)}{2 a_{1}}+t\right)\right\}+O\left(\frac{1}{\rho_{n}^{2}}\right) .
\end{gathered}
$$

For nodal points of $\omega_{2}\left(t, \rho_{n}\right)$, again, taking $\sin \frac{\rho_{n}}{a_{2}}\left(\frac{\pi\left(a_{2}-a_{1}\right)}{2 a_{1}}+t\right) \neq 0$ into account for sufficiently large $n$, we get

$$
\begin{aligned}
& \cot \frac{\rho_{n}}{a_{2}}\left(\frac{\pi\left(a_{2}-a_{1}\right)}{2 a_{1}}+t\right)\left[1-\frac{\left(A\left(\frac{\pi}{2}, \rho_{n}, \theta\right)+C\left(t, \rho_{n}, \theta\right)\right)\left(a_{1}+a_{2}\right)}{2 \rho_{n} a_{1} a_{2}}\right] \\
= & \frac{\left(a_{1}+a_{2}\right)\left(B\left(\frac{\pi}{2}, \rho_{n}, \theta\right)+D\left(t, \rho_{n}, \theta\right)\right)}{2 \rho_{n} a_{1} a_{2}}+O\left(\frac{1}{\rho_{n}^{2}}\right) .
\end{aligned}
$$

and thus

$$
\begin{aligned}
& \tan \left(\frac{\rho_{n}}{a_{2}}\left(\frac{\pi\left(a_{2}-a_{1}\right)}{2 a_{1}}+t\right)+\frac{\pi}{2}\right) \\
= & -\frac{\left(a_{1}+a_{2}\right)\left(B\left(\frac{\pi}{2}, \rho_{n}, \theta\right)+D\left(t, \rho_{n}, \theta\right)\right)}{2 \rho_{n} a_{1} a_{2}}+O\left(\frac{1}{\rho_{n}^{2}}\right) .
\end{aligned}
$$

Thus, solving the equation (23), one obtains

$$
\begin{gathered}
t_{n}^{j}=-\frac{\pi\left(a_{2}-a_{1}\right)}{2 a_{1}}+\frac{\left(j-\frac{1}{2}\right) \pi a_{2}}{\rho_{n}} \\
-\frac{\left(a_{1}+a_{2}\right)\left(B\left(\frac{\pi}{2}, \rho_{n}, \theta\right)+D\left(t_{n}^{j}, \rho_{n}, \theta\right)\right)}{2 \rho_{n}^{2} a_{1}}+O\left(\frac{1}{\rho_{n}^{3}}\right) .
\end{gathered}
$$

Substituting (20) and (21) into (24) we have

$$
t_{n}^{j}=-\frac{\pi\left(a_{2}-a_{1}\right)}{2 a_{1}}+\frac{\left(j-\frac{1}{2}\right) \pi a_{2}}{\rho_{n}^{0}}
$$




$$
\begin{gathered}
-\frac{\left(j-\frac{1}{2}\right) a_{2}\left[\frac{a_{1}+a_{2}}{2 a_{1} a_{2}}\left(B\left(\frac{\pi}{2}, \rho_{n}^{0}, \theta\right)+D\left(\pi, \rho_{n}^{0}, \theta\right)\right)\right]}{\left(\rho_{n}^{0}\right)^{3}} \\
-\frac{\left.\left(a_{1}+a_{2}\right)\left[B\left(\frac{\pi}{2}, \rho_{n}^{0}, \theta\right)+D\left(\frac{\left(j-\frac{1}{2}\right) \pi a_{2}}{\rho_{n}^{0}}, \rho_{n}^{0}, \theta\right)\right)\right]}{2\left(\rho_{n}^{0}\right)^{2} a_{1}}+O\left(\frac{1}{n^{3}}\right), \quad j=\overline{\left[\frac{n}{2}\right]+1, n .}
\end{gathered}
$$

Thus we have proven the following theorem:

Theorem 4.1. For sufficiently large $n$, we have the formulas (22) and (25) of the nodal points for the problem (1)-(5).

\section{REFERENCES}

[1]. Norkin S.B., Differential equations of the second order with retarded argument, Translations of Mathematical Monographs, AMS, Providence, RI, 1972.

[2]. Mukhtarov O.Sh., Kadakal M., Muhtarov F.S., On discontinuous Sturm-Liouville problems with transmission conditions, J. Math. Kyoto Univ., 44-4 (2004) 779-798.

[3]. Mukhtarov O.Sh., Tunç E., Eigenvalue problems for Sturm--Liouville equations with transmission conditions, Israel J. Math. 144 (2004) 367--380.

[4]. Aydemir K., Mukhtarov O.Sh., Class of Sturm-Liouville problems with eigenparameter dependent transmission conditions, Numer. Funct. Anal. Optim., 38-10 (2017) 1260-1275.

[5]. Çakmak Y., Keskin B., Uniqueness theorems for Sturm-Liouville operator with parameter dependent boundary conditions and finite number of transmission conditions, Cumhuriyet Sci. J., 38-3 (2017) 535-543.

[6]. Kandemir M., Asymptotic distribution of eigenvalues for fourth-order boundary-value problem with discontinuous coefficients and transmission conditions, Commun. Fac. Sci. Univ. Ank. Ser. A1 Math. Stat., 66-1 (2017) 133-152.

[7]. Li K., Sun J., Hao X., Weyl function of Sturm-Liouville problems with transmission conditions at finite interior points, Mediter. J. Math., 14-189 (2017) 1-15.

[8]. Uğurlu E., Taş K., A new method for dissipative dynamic operator with transmission conditions, Complex Anal. Oper. Theory, 12 (2018) 1027-1055.

[9]. Zettl A., Adjoint and self-adjoint boundary value problems with interface conditions, SIAM J. Appl. Math., 16 (1968) 851-859.

[10]. Buschmann D., Stolz G., Weidmann J., One-dimensional Schrödinger operators with local point interactions, J. Reine Angew. Math., 467 (1995) 169-186.

[11]. Gelfand I.M., Levitan B.M., On a formula for eigenvalues of a differential operator of second order, Doklady Akademii Nauk SSSR, 88 (1953) 593--596 (Russian).

[12]. Dikii L.A., Trace formulas for Sturm-Liouville differential equations, Uspekhi Mat. Nauk, 12-3 (1958) 111-143.

[13]. Maksudov F.G., Bayramoglu M., Adıguzelov E., On a regularized traces of the Sturm-Liouville operator on a finite interval with the unbounded operator coefficient, Doklady Akademii Nauk SSSR, 277-4 (1984); English translation: Soviet Math. Dokl., 30 (1984) 169-173.

[14]. Bayramoglu M., Sahinturk H., Higher order regularized trace formula for the regular SturmLiouville equation contained spectral parameter in the boundary condition, Appl. Math. Comput., 186 (2007) 1591-1599.

[15]. Gül E., On the regularized trace of a second order differential operator, Appl. Math. Comput., 198 (2008) 471-480. 
[16]. Yang C-F., Trace and inverse problem of a discontinuous Sturm-Liouville operator with retarded argument, J. Math. Anal. Appl., 395 (2012) 30-41.

[17]. Bayramoglu M., Bayramov A., Şen E., A regularized trace formula for a discontinuous SturmLiouville operator with delayed argument, Electronic Journal of Differential Equations, 2017-104 (2017) $1-12$.

[18]. Hira F., A trace formula for the Sturm-Liouville type equation with retarded argument, Commun. Fac. Sci. Univ. Ank. Ser. A1 Math. Stat., 66-1 (2017) 124-132.

[19]. Keskin B., Ozkan A.S., Inverse nodal problems for impulsive Sturm-Liouville equation with boundary conditions depending on the parameter, Advances in Analysis, 2-3 (2017) 151-156.

[20]. Keskin B., Reconstruction of the Volterra-type integro-differential operator from nodal points, Boundary Value Problems, 2018-47 (2018) 1-8.

[21]. Keskin B., Ozkan A.S., Inverse nodal problems for Dirac-type integro-differential operators, J. Differ. Eq., 263 (2017) 8838-8847.

[22]. Bayramov A., Çalışkan S., Uslu S., Computation of eigenvalues and eigenfunctions of a discontinuous boundary value problem with retarded argument, Appl. Math. Comput., 191 (2007) 592-600.

[23]. Şen E., Bayramov A., Calculation of eigenvalues and eigenfunctions of a discontinuous boundary value problem with retarded argument which contains a spectral parameter in the boundary condition, Math. Comput. Model., 54-(11-12) (2011) 3090-3097.

[24]. Yurko V.A., Inverse Spectral Problems for Differential Operators and Their Applications, Gordon and Breach, Amsterdam, 2000. 